\title{
MAXIMAL FUNCTIONS OF PLURISUBHARMONIC FUNCTIONS
}

\author{
By
}

\author{
Hong Oh KIM and Yeon Yong PARK
}

\begin{abstract}
We show that for nonnegative plurisubharmonic functions on the unit ball of $C^{n}$ the admissible maximal functions are dominated by the radial maximal functions in $L^{p}$-mean. This gives another characterization of the class $M^{p}$ of holomorphic functions and its invariance under the compositions by automorphisms of the unit ball. As a consequence of the invariance all onto endomorphisms of $M^{1}$ $(n=1)$ are characterized.
\end{abstract}

\section{Introduction.}

Let $B$ be the unit ball of $C^{n}(n \geqq 1)$ and let $\sigma$ denote the Lebesgue measure on $S=\partial B$, normalized so that $\sigma(S)=1$. For a function $u: B \rightarrow C$, the radial maximal function $\mathscr{M u}$ on $S$ is defined by

$$
\mathscr{M} u(\eta)=\sup \{|u(r \eta)|: 0 \leqq r<1\}, \quad \eta \in S .
$$

For $\alpha>1$ and $\eta \in S$, we let

$$
D_{\alpha}(\eta)=\left\{z \in B:|1-<z, \eta>|<\frac{\alpha}{2}\left(1-|z|^{2}\right)\right\} .
$$

The admissible maximal function $\mathscr{M}_{\alpha} u$ on $S$ is defined by

$$
\mathscr{M}_{\alpha} u(\eta)=\sup \left\{|u(z)|: z \in D_{\alpha}(\eta)\right\} .
$$

We prove the following theorem.

THEOREM I. For $0<p<\infty$, there is a positive constant $C=C(n, p, \alpha)$ such that if $u \geqq 0$ is plurisubharmonic in $B$ then

$$
\int_{S} \mathscr{M}_{\alpha} u(\eta)^{p} d \sigma(\eta) \leqq C \int_{S} \mathscr{M} u(\eta)^{p} d \sigma(\eta)
$$

For $n=1$, the corresponding theorem for harmonic functions on the upper half plane appears in [3, Theorem 3.6].

1980 Mathematics Subject Classification (1985 Revision). 31C10, 32 A35.

Key words and phrases. plurisubharmonic function, maximal function; class $M^{p}$.

Received March 4, 1991, Revised June 4, 1991. 
For an application of Theorem I, we consider the class $M^{p}(B)(0<p<\infty)$ of holomorphic functions $f$ on $B$ for which

$$
\int_{S}\left(\log { }^{+} \mathscr{M} f(\eta)\right)^{p} d \sigma(\eta)<\infty
$$

For $n=1$, these classes as topological algebras have been studied in $[7,10]$ for $p>1$ and in $[2,5,6]$ for $p=1$. For $n \geqq 1$, it is shown in [2] that

$$
\bigcup_{p>0} H^{p} \subsetneq \bigcap_{p>1} M^{p} \subsetneq M^{1} \subsetneq N^{+},
$$

where $H^{p}$ is the usual Hardy space and $N^{+}$is the Smirnov class on $B$. The main theorem of [2] concerns with the boundary behavior of functions in the class $M^{p}(p \geqq 1)$, with its application to outer factors of functions in $M^{1}$ when $n=1$.

If we take $u=\log ^{+}|f|$ with holomorphic functions $f$ on $B$ in Theorem I, we get the following characterization of $M^{p}$ immediately.

THEOREM II. A holomorphic function $f$ on $B$ belongs to $M^{p}$ if and only if

$$
\int_{S}\left(\log ^{+} \mathscr{M}_{\alpha} f(\eta)\right)^{p} d \sigma(\eta)<\infty
$$

Since every automorphism of $B$ maps any radius into a curve which approaches the boundary nontangentially, the following corollary is immediate.

COROLLARY III. The class $M^{p}(0<p<\infty)$ is invariant under the compositions of automorphisms of $B$.

When $p>1$, this fact is not new because $M^{p}(p>1)$ can be defined by means of boundary functions. See $[2,7]$. As a consequence of this corollary we can characterize all onto algebra endomorphisms of $M^{1}$ for the case $n=1$. For the case $p>1$, see [7].

TheOREM IV. Let $n=1$. Then $\Gamma: M^{1} \rightarrow M^{1}$ is an onto algebra endomorphism if and only if

$$
\Gamma(f)=f \circ \varphi, \quad f \in M^{1}
$$

for some automorphism $\varphi$ of the unit disc $U$ of $C^{1}$. In particular, $\Gamma$ is invertible in this case and $\Gamma^{-1}(f)=f \circ \varphi^{-1}, f \in M^{1}$.

The proof will be given in the last section. The theorem might be true for $n>1$ but we do not have a proof. 


\section{An inequality of Hardy and Littlewood.}

The following lemma is due to Hardy and Littlewood. It is stated in $[3,4]$ for $|u|$ with harmonic functions $u$ but the proof is exactly the same for nonnegative subharmonic functions.

2.1. LemmA. If $u \geqq 0$ is subharmonic on the disc $D\left(z_{0}, R\right)$ with center at $z_{0}$ and radius $R>0$ in the complex plane $C$ and if $0<p<\infty$, then

$$
u\left(z_{0}\right) \leqq K\left(\frac{1}{\pi R^{2}} \iint_{D\left(z_{0}, R\right)} u(z)^{p} d x d y\right)^{1 / p}
$$

where $K=K(p)$ is a positive constant independent of $u$.

The next lemma will be a polydisc version of the above inequality. Its statement is suitably adapted for the proof of Theorem I.

Let $z=r \zeta \in B$ and $R>0$. Let $\zeta_{2}, \cdots, \zeta_{n} \in S$ be such that $\zeta_{2}, \zeta_{2}, \cdots, \zeta_{n}$ form an orthonomal basis for $C^{n}$. Define a polydisc $\Delta(z, R)$ with respect to the basis $\zeta, \zeta_{2}, \cdots, \zeta_{n}$ at $z$ as follows :

$$
\begin{aligned}
\Delta(z, R) & \equiv \Delta\left(z, R ; \zeta, \zeta_{2}, \cdots, \zeta_{n}\right) \\
& =\left\{w=z+\lambda \zeta+\sum_{2}^{n} \lambda_{j} \zeta_{j}:|\lambda|<R,\left|\lambda_{j}\right|<R^{1 / 2}, 2 \leqq j \leqq n\right\} .
\end{aligned}
$$

2.2. LEMMA. Let $\Delta=\Delta(z, R) \subset B$. If $u \geqq 0$ is plurisubharmonic in $B$ and $0<p<\infty$, then

$$
u(z)^{p} \leqq K \frac{1}{m_{n}(\Delta)} \int_{\Delta} u(w)^{p} d m_{n}(w),
$$

where $K=K(n, p)$ is a positive constant independent of $u$ and $d m_{n}$ is the Lebesgue measure on $C^{n}$.

Proof. We define

$$
v\left(\lambda, \lambda_{2}, \cdots, \lambda_{n}\right)=u\left(z+\lambda \zeta+\lambda_{2} \zeta_{2}+\cdots+\lambda_{n} \zeta_{n}\right) .
$$

Since $u$ is plurisubharmonic in $B, v$ is an $n$-subharmonic function for $|\lambda|<R$, $\left|\lambda_{j}\right|<R^{1 / 2}(2 \leqq j \leqq n)$. We now apply Lemma $2.1 n$ times to $v$. The positive constants $K$ 's in the following are not the same in each occurence but are independent of $v$.

$$
\begin{aligned}
v(0, \cdots, 0)^{p} & \leqq K \frac{1}{R} \int_{\left|\lambda_{n}\right|<R^{1 / 2}} v\left(0, \cdots, 0, \lambda_{n}\right) d m_{1}\left(\lambda_{n}\right) \\
& \leqq \cdots \\
& \leqq K \frac{1}{R^{n-1}} \int \cdots \int_{\mid \lambda_{j \mid<R^{1 / 2}(2 \leq j \leq n)} v\left(0, \lambda_{2}, \cdots, \lambda_{n}\right)^{p} d m_{1}\left(\lambda_{2}\right) \cdots d m_{1}\left(\lambda_{n}\right)}
\end{aligned}
$$




$$
\leqq K \frac{1}{R^{n+1}} \int \cdots \int_{\left|\lambda_{1}\right|<R \cdot\left|\lambda_{j}\right|<R^{1 / 2}(2 \leq j \leq n)} v\left(\lambda_{1}, \cdots, \lambda_{n}\right)^{p} d m_{1}\left(\lambda_{1}\right) \cdots d m_{1}\left(\lambda_{n}\right) .
$$

Therefore, we have

$$
u(z)^{p} \leqq K \frac{1}{m_{n}(\Delta)} \int_{\Delta} u(w)^{p} d m_{n}(w) . \quad \text { Q. E. D. }
$$

\section{Geometric lemmas.}

3.1. LemMA. Let $z=r \zeta \in B$ and let $\Delta\left(z, \varepsilon\left(1-r^{2}\right)\right) \subset B$ for a choice of $\zeta_{2}, \cdots$, $\zeta_{n} \in S$ and $\varepsilon>0$. If $r>1 / 2$ and $w \in \Delta\left(z, \varepsilon\left(1-r^{2}\right)\right)$ then

$$
r-\delta\left(1-r^{2}\right)<|w|<r+\delta\left(1-r^{2}\right)
$$

for some choice of a positive constant $\delta=\delta(n, \varepsilon)$ independent of $z$ and $\zeta$ 's.

ProOF. Suppose $w=z+\lambda \zeta+\sum_{2}^{n} \lambda_{j} \zeta_{j} \in \Delta\left(z ; \varepsilon\left(1-r^{2}\right)\right)$. Then

$$
\begin{aligned}
|w|^{2} & =|r+\lambda|^{2}+\sum_{2}^{n}\left|\lambda_{j}\right|^{2} \leqq r^{2}+|\lambda|^{2}+2|\lambda|+(n-1) \varepsilon\left(1-r^{2}\right) \\
& \leqq r^{2}+(n+2) \varepsilon\left(1-r^{2}\right) .
\end{aligned}
$$

Also,

If $r>1 / 2$ then

$$
\begin{aligned}
|w|^{2} & \geqq(r-|\lambda|)^{2}=r^{2}-2 r|\lambda|+|\lambda|^{2} \\
& \geqq r^{2}-2|\lambda| \geqq r^{2}-2 \varepsilon\left(1-r^{2}\right) .
\end{aligned}
$$

$$
|| w|-r| \leqq\left. 2|| w\right|^{2}-r^{2} \mid \leqq 2(n+2) \varepsilon\left(1-r^{2}\right) .
$$

So we can take $\delta=2(n+2) \varepsilon$.

Q.E.D.

The following lemma appears in [1] but its proof is included for the sake of completeness.

3.2. Lemma. Let $\beta>\alpha>1$ and $z=r \zeta \in D_{\alpha}(\eta)$. Then there is a positive constant $\varepsilon=\varepsilon(n, \alpha, \beta)$ such that

$$
\Delta\left(z, \varepsilon\left(1-r^{2}\right)\right) \subset D_{\beta}(\eta)
$$

for any choice of $\zeta_{2}, \cdots, \zeta_{n} \in S$.

Proof. Suppose $w=z+\lambda \zeta+\sum_{2}^{n} \lambda_{j} \zeta_{j} \in \Delta\left(z, \varepsilon\left(1-r^{2}\right)\right)$. Then $|\lambda|<\varepsilon\left(1-r^{2}\right)$ and and $\left|\lambda_{j}\right|<\left\{\varepsilon\left(1-r^{2}\right)\right\}^{1 / 2}$. By the orthogonality of $\zeta$ and $\zeta_{j}$, the Schwarz lemma and the hypothesis $z \in D_{\alpha}(\eta)$, we have

$$
\begin{aligned}
\left|\left\langle\zeta_{j}, \eta\right\rangle\right| & =\left|\left\langle\zeta_{j}, \eta-r \zeta_{j}\right\rangle\right| \\
& \leqq|\eta-r \zeta|
\end{aligned}
$$


We compute

$$
\begin{aligned}
& \leqq \sqrt{2}|1-\langle r \zeta, \eta\rangle|^{1 / 2} \\
& \leqq\left\{\alpha\left(1-r^{2}\right)\right\}^{1 / 2} .
\end{aligned}
$$

$$
\begin{aligned}
|1-\langle w, \eta\rangle| & =\left|1-\left(\langle r \zeta, \eta\rangle+\lambda\langle\zeta, \eta\rangle+\sum_{2}^{n} \lambda_{j}\left\langle\zeta_{j}, \eta\right\rangle\right)\right| \\
& \leqq \frac{\alpha}{2}\left(1-r^{2}\right)+\varepsilon\left(1-r^{2}\right)+\sum_{2}^{n}\left\{\varepsilon\left(1-r^{2}\right)\right\}^{1 / 2}\left|\left\langle\zeta_{j}, \eta\right\rangle\right| \\
& \leqq\left\{\frac{\alpha}{2}+\varepsilon+(n-1) \varepsilon^{1 / 2} \alpha^{1 / 2}\right\}\left(1-r^{2}\right)
\end{aligned}
$$

On the other hand, from the proof of Lemma 3.1, we have

$$
1-|w|^{2} \geqq\{1-(n+2) \varepsilon\}\left(1-r^{2}\right) .
$$

Therefore we can choose $\varepsilon=\varepsilon(n, \alpha, \beta)>0$ so small that

$$
|1-\langle w, \eta\rangle|<\frac{\beta}{2}\left(1-|w|^{2}\right),
$$

for any $w \in \Delta\left(z, \varepsilon\left(1-r^{2}\right)\right)$. Therefore $\Delta\left(z, \varepsilon\left(1-r^{2}\right)\right) \subset D_{\beta}(\eta)$.

Q.E.D.

We define the radial projection $\pi$ from $B \backslash\{0\}$ onto $S$ as

$$
\pi(w)=w /|w|, \quad w \in B \backslash\{0\} .
$$

For $\eta \in S$ and $\delta>0$,

$$
Q(\eta, \delta)=\{\boldsymbol{\zeta} \in S:|1-\langle\zeta, \eta\rangle|<\delta\}
$$

is the nonisotropic "ball" of radius $\delta^{1 / 2}$ around $\eta$. The volume $\sigma(Q(\eta, \delta))$ is roughly propotional to $\delta^{n}$, i.e., $\sigma(Q(\eta, \delta)) \approx \delta^{n}$. See [9, Proposition 5.1.4].

33. LemmA. Let $z=r \zeta \in D_{\alpha}(\eta), r>0$ and $\beta>\alpha>1$. Then there is a positive constant $\varepsilon=\varepsilon(n, \alpha, \beta)$ so small that

$$
\pi\left(\Delta\left(z, \varepsilon\left(1-r^{2}\right)\right) \subset Q\left(\eta,\left(\frac{\beta}{2}+1\right)\left(1-r^{2}\right)\right)\right.
$$

for any choice of $\zeta_{2}, \cdots, \zeta_{n}$.

Proof. Chooce $\beta^{\prime}$ so that $\beta>\beta^{\prime}>\alpha$. Let $w=\rho w \in \Delta\left(z, \varepsilon\left(1-\gamma^{2}\right)\right)$. Then

$$
\begin{aligned}
|1-\langle\omega, \eta\rangle| & =|1-\langle\rho \omega, \eta\rangle-(1-\rho)\langle\omega, \eta\rangle| \\
& \leqq|1-\langle\omega, \eta\rangle|+\left(1-\rho^{2}\right) .
\end{aligned}
$$

By Lemma 3.2 , we can choose $\varepsilon>0$ so small that

$$
|1-\langle w, \eta\rangle|<\frac{\beta^{\prime}}{2}\left(1-r^{2}\right) .
$$


From the proof of Lemma 3.1, we have

$$
1-\rho^{2} \leqq(1+2 \varepsilon)\left(1-r^{2}\right) \text {. }
$$

Therefore we have

$$
|1-\langle\omega, \eta\rangle|<\left(\frac{\beta^{\prime}}{2}+1+2 \varepsilon\right)\left(1-r^{2}\right) \text {. }
$$

If we choose $\varepsilon=\varepsilon(n, \alpha, \beta)>0$ even smaller so that $\beta^{\prime} / 2+1+2 \varepsilon<\beta / 2+1$, we have

$$
|1-\langle\omega, \eta\rangle|<\left(\frac{\beta}{2}+1\right)\left(1-r^{2}\right) \text {; }
$$

so that $\omega \in Q\left(\eta,(\beta / 2+1)\left(1-r^{2}\right)\right)$.

Q.E.D.

\section{Proof of Theorem I.}

It suffices to prove the theorem for a modified admissible maximal function (with the same notation) as

$$
\mathscr{M}_{\alpha} u(\eta)=\sup \left\{|u(z)|:|z| \geqq \frac{1}{2}, z \in D_{\alpha}(\eta)\right\} .
$$

Let $z=r \zeta \in D_{\alpha}(\eta), r \geqq 1 / 2$ and $\beta>\alpha$. By Lemmas 3.1, 3.2 and 3.3, we can choose positive constants $\varepsilon=\varepsilon(n, \alpha, \beta)$ and $\delta=\delta(n, \varepsilon)=\delta(n, \alpha, \beta)$ so that

(i) $\Delta=\Delta\left(z, \varepsilon\left(1-r^{2}\right)\right) \subset D_{\beta}(\eta)$ for a choice of $\zeta_{2}, \cdots, \zeta_{n}$,

(ii) $\pi(\Delta) \subset Q\left(\eta,(\beta / 2+1)\left(1-r^{2}\right)\right)$,

(iii) $r-\delta\left(1-r^{2}\right)<|w|<r+\delta\left(1-r^{2}\right)$ if $w \in \Delta$.

Using Lemma 2.2, we have the following computation in which the constants $K=K(n, p, \delta)$ are not the same in each occurrence, but are independent of $u$.

$$
\begin{aligned}
u(z)^{p / 2} & \leqq K \frac{1}{\left(1-r^{2}\right)^{n+1}} \int_{\Delta} u(w)^{p / 2} d m_{n}(w) \\
& \leqq K \frac{1}{\left(1-r^{2}\right)^{n+1}} \int_{r-\delta\left(1-r^{2}\right)}^{r+\delta\left(1-r^{2}\right)} \rho^{2 n-1} d \rho \int_{Q\left(\eta,(\beta / 2+1)\left(1-r^{2}\right)\right)} \mathscr{M} u(\omega)^{p / 2} d \sigma(\omega) \\
& \leqq K \frac{1}{\left(1-r^{2}\right)^{n}} \int_{Q} \mathscr{M} u(\omega)^{p / 2} d \sigma(\omega) \\
& \leqq K \frac{1}{\sigma(Q)} \int_{Q} \mathscr{M} u(\omega)^{p / 2} d \sigma(\omega) \\
& \leqq K M\left\{(\mathscr{M} u)^{p / 2}\right\}(\eta),
\end{aligned}
$$

where $M$ is the Hardy-Littlewood maximal function operator on $S$. Therefore we have

$$
\left\{\mathscr{M}_{\alpha} u(\eta)\right\}^{p / 2} \leqq K M\left\{(\mathscr{M} u)^{p / 2}\right\}(\eta) .
$$

We note that the constan $K$ is eventally dependent on $n, p, \alpha$ from the choice 
of $\beta$ and $\delta$. By the Hardy-Littlewood maximal theorem [9, Theorem 5.2.6], we have

$$
\int_{S} \mathscr{M}_{\alpha} u(\eta)^{p} d \sigma(\eta) \leqq C \int_{S} \mathscr{M} u(\eta)^{p} d \sigma(\eta)
$$

for some positive constant $C=C(n, p, \alpha)$ indpendent of $u$.

Q.E.D.

\section{Proof of Theorem IV.}

By corollary III, every automorphism $\varphi$ of $U$ defines an algebra isomorphism $\Gamma(f)=f \circ \varphi, \varphi \in M^{1}$. Conversely, let $\Gamma$ be any onto endomorphism of $M^{1}$. We will follow the corresponding proof for the case $N^{+}[8]$. Let $\varphi=\Gamma(z)$ and let $\lambda=U . \quad(z$ denotes the identity function on $U$.$) Define \gamma(f)=\Gamma(f)(\lambda), f \in M^{1}$. Since $\gamma$ is a multiplicative linear functional on $M^{1}, \gamma$ corresponds to the point evaluation at some $\beta \in U$ by Theorem 6.4 of [6]. Thus $\beta=\gamma(z)=\Gamma(z)(\lambda)=\varphi(\lambda)$. Hence $\varphi(\lambda) \in U$ for all $\lambda \in U$ and $\Gamma(f)(\lambda)=f(\varphi(\lambda)), f \in M^{1}, \lambda \in U$. Since $\Gamma$ is onto, $\varphi$ is not constant. Thus $\varphi(U)$ is open in $U$. Therefore $\Gamma$ is one-to-one (and onto). Thus $\Gamma^{-1}$ is also an onto endomorphism, so $\Gamma^{-1}(f)=f \circ \psi, f \in M^{1}$, for some holomorphic self-map $\phi$ of $U$. But then $z=\Gamma \Gamma^{-1}(z)=\Gamma(\psi)=\psi \circ \varphi$ and $\varphi \circ \psi=z$. Therefore $\varphi$ is an automorphism of $U$.

Q.E.D.

\section{Acknowledgement.}

The first author wishes to express his gratitude to Professor P. Ahern for the valuable discussions. $\mathrm{He}$ is in part supported by TGRC (KOSEF).

\section{References}

[1] P.R. Ahern and J. Bruna, Maximal and area integral characterization of HardySobolev space in the unit ball of $C^{n}$, Rivista Matemática Iberoamericana, 4 (1988), 123-153.

[2] B.R. Choe and H.O. Kim, On the boundary behavior of functions holomorphic on the ball, to appear in Complex Variables.

[3] J.B. Garnett, Bounded analytic functions, Academic Press, New York, 1981.

[4] G.H. Hardy and J.E. Littlewood, Some properties of conjugate functions, J. Reine Angew. Math. 167 (1932), 405-423.

[5] H.O. Kim, On closed maximal ideals of $M$, Proc. Japan Academy, 62A (1986), 343-346.

[6] H.O. Kim, On an $F$-algebra of holomorphic functions, Can. J. Math., 40 (1988), 718-741.

[7] N. Mochizuki, Algebras of holomorphic functions between $H^{p}$ and $N_{*}$, Proc. AMS, 105 (1989), 898-902.

[8] J.W. Roberts and M. Stoll, Prime and principal ideals in the algebra $N^{+}$, Arch. Math., 27 (1976), 387-393.

[9] W. Rudin, Function theory in the unit ball of $C^{n}$, Springer-Verlag, New York, 1980. 
[10] M. Stoll, Mean growth and Taylor coefficients of some topological algebras of analytic functions, Ann. Polon. Math., 35 (1977), 139-158.

Department of Mathematics

Korea Advanced Institute

of Science and Technology

Taejon 305-701, Korea 